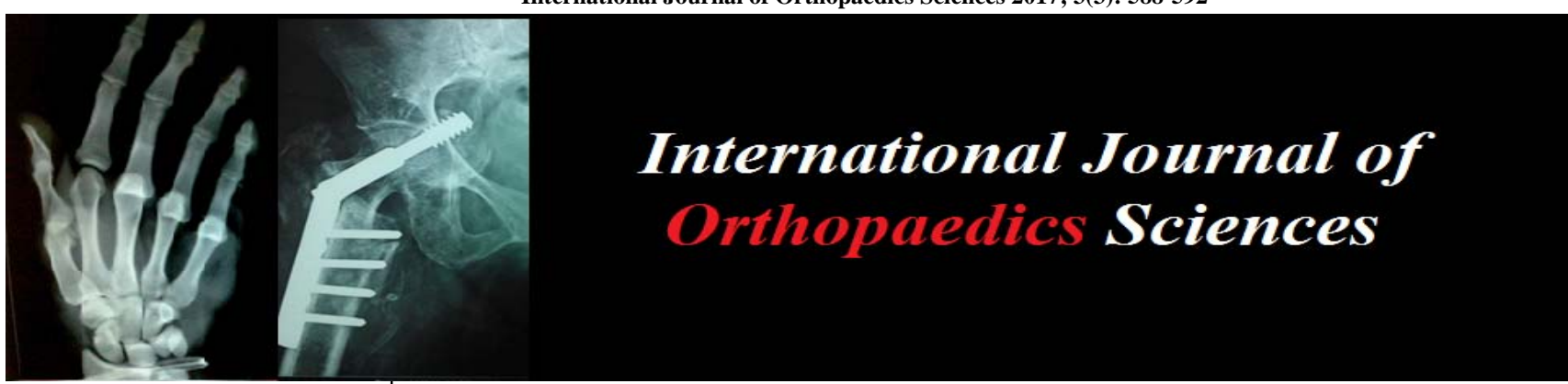

ISSN: $2395-1958$

IJOS 2017; 3(3): 588-592

(C) 2017 IJOS

www.orthopaper.com

Received: 23-05-2017

Accepted: 24-06-2017

Dr. Anas M

MS(Orthopaedics) Senior

Resident Department of

Orthopaedics, Govt: Medical

College, Thiruvananthapuram,

Kerala, India

Dr. Ansari N

MS (Orthopaedics) Assistant

Professor Department of

Orthopaedics, Govt: Medical

College, Thiruvananthapuram,

Kerala, India
Correspondence

Dr. Ansari N

MS (Orthopaedics) Assistant

Professor Department of

Orthopaedics, Govt: Medical

College, Thiruvananthapuram,

Kerala, India

\section{Functional outcome of minimally invasive reconstruction of medial patello femoral ligament using Quadricep tendon graft in recurrent patellofemoral instability}

\section{Dr. Anas M and Dr. Ansari N}

DOI: http://dx.doi.org/10.22271/ortho.2017.v3.i3i.93

Abstract

Recurrent patellofemoral instability is a chronic often painful condition most commonly affecting females of 10-17 years. Aim of the study was to evaluate the functional outcome of minimally invasive reconstruction of medial patello femoral ligament using Quadricep tendon graft in recurrent patellofemoral instability. 16 cases of recurrent patellar instability treated in a tertiary care hospital from February 2015 to march 2016 were included. All cases undergone surgery by same team after history, physical examination and radiological imaging. After post operative rehabilitation schedule, all were followed up to 8 months and functional outcome was evaluated using Kujala Anterior Knee Pain Scale (AKPS). The mean Q angle was 22.25. Among 16 cases 12 showed congruence angle greater than -6.13 out of 16 showed a positive basett's sign. Apprehension and patellar glide test was positive in all cases. The mean Kujala knee score before surgery was 62.50 (range 56-66) out of which $25 \%$ of cases showed poor and $75 \%$ with fair scores. The mean Kujala score 8 months after surgery was 86 . (range 78-97). $12.5 \%$ of cases showed excellent outcome $37.5 \%$ were having good and cases with fair outcome being $50 \%$. Minimally invasive quadriceps tendon graft for medial patellofemoral ligament reconstruction for chronic patellofemoral instability showed significant improvement in Kujala knee score ( $\mathrm{P}$ value $<0.001)$. The simple, easily reproducible and novel technique of minimally invasive quadriceps tendon graft for medial patellofemoral ligament reconstruction proves to be having good functional out come in recurrent patellar instability.

Keywords: Recurrent patellofemoral instability, Medial patello femoral ligament (MPFL), Quadricep tendon graft, Bassett's sign, Q angle. Kujala Anterior Knee Pain Scale (AKPS), The Beighton hypermobility score, Insal salvetti ratio

\section{Introduction}

Recurrent patellofemoral instability a chronic painful condition following dislocation of patella affects 5.8 per 100,000 individuals and is more prevalent among females aged 10-17 years ${ }^{[1]}$. It is estimated that there is a seven times risk of recurrence following a single dislocation and $50 \%$ chance after second episode ${ }^{[3]}$. Chronic knee pain is very often in this condition due to articular cartilage damage ${ }^{[2]}$. Ligamentous laxity, genu valgum, femoral anteversion, excessive tibial torsion, trochlear dysplasia, patella alta, and insufficiency or rupture of the medial patellofemoral ligament (MPFL) are etiological factors regarding recurrent patellofemoral instability. Eventhough surgical intervention is required to manage instability, there is no concesus regarding the optimal technique among the many ones described based on reducing the lateral displacement which is achieved either by lateral release or medial reafing.

The medial patellofemoral ligament (MPFL), the primary passive restraint to patellar lateral displacement is a $55 \mathrm{~cm}$ long band of retinacular tissue connecting the femoral medial epicondyle to the medial edge of the patella ${ }^{[4]}$ and its reconstruction using either semitendinosus or gracilis or quadriceps is becoming popular now a days. The technique of MPFL reconstruction using quadriceps has advantage of morphological similarity and no tunnel or anchors in patella. 


\section{Materials and Method}

We performed a single center prospective cohort study of 16 patients with chronic patellar instability treated with minimally invasive reconstruction of medial patello femoral ligament using a Quadricep tendon graft at a tertiary care hospital from February 2015 to march 2016. Chronic patellar instability is evaluated by history, clinical examination and imaging. A history for more than 2 time lateral displacement is included in the study. Cases were examined Q angle, The Beighton hypermobility score, passive patellar glide test, patellar tracking and $\mathrm{j}$ sign, lateral tilt, apprehension test Bassett's sign. Imaging included weight bearing anteroposterior (AP), posteroanterior (PA) ( $45^{\circ}$ flexion), and true lateral $\left(30^{\circ}\right.$ flexion) radiographs of the knee. Cases with diagnosed neuromuscular disorders and myopathies and congenital muscular disorders were excluded from the study. $\mathrm{Q}$ angle is the angle between a line drawn from the anterior superior iliac spine to the center of the patella and line from the center of the patella to the tibial tubercle. A large Q angle indicates the lateral directed force of extensor mechanism that predisposes the lateral dislocation of patella. The Beighton hypermobility score asses overall generalized ligamentous laxity which involves a 9-point scale on which a score greater than 5 indicates hypermobility; one point is assigned for each of the following findings: passive hyperextension of each small finger $>90^{\circ}$, passive abduction of each thumb to the volar/radial surface of the forearm, hyperextension of each elbow $>10^{\circ}$, hyperextension of each knee $>10^{\circ}$, forward flexion of the trunk with the ability to place both palms flat on the floor with full knee extension

The passive patellar glide test is a simple manoeuver to evaluate for patellar hypermobility and a glide longer than the width of three quadrants is consistent with hypermobility.

Bassett's sign indicate a rupture of the MPFL; it consists of pain with palpation of the adductor tubercle, which is the femoral attachment of the MPFL.

Functional outcome was evaluated by using Kujala Anterior Knee Pain Scale (AKPS) 8 months after surgery ${ }^{[12]}$. The AKPS is an established self-report questionnaire to evaluate patients with disorders of the knee. It consists of 13 items documenting response to six activities causing knee complaints (walking, running, jumping, climbing stairs, squatting, sitting with bended knees) and associated symptoms such as limitation of range of motion, limp and swelling. The maximum score was 100.Lower score indicates poor prognosis ${ }^{[13]}$. A score $<64$ is considered to be a poor result, 65 to 84 a fair, 85 to 94 a good and $>94$ an excellent result ${ }^{[14]}$.

\section{Surgical Technique \\ Positioning}

Patient is positioned supine in table. with a pillow is kept under the knee to keep flexed and passive full extension is possible, scrubbed painted and draped.

\section{Surgical Steps}

With knee flexion of 90, a 3-cm transverse skin incision is made over the superomedial pole of the patella. After incising prepatellar bursa, the patellar aponeurosis is ${ }^{[11]}$ exposed. A Langenbeck retractor is introduced. Quadriceps is subcutaneously exposed proximal to the patella.

An open tendon stripper is introduced at the middle of superior patellar border and pushed distally minimum upto $8 \mathrm{~cm}$.thickness of graft is determined by tendon separator. Distal end of graft is freed through an incision made distally.
By use of web-stitch technique with 1-0 prolene, free proximal end is sutured.

A $1.5-\mathrm{cm}$ skin incision is made over the adductor tubercle. Prepatellar tissue is elevated, creating a tunnel that reaches the medial edge of the graft using a periosteal elevator. A curved clamp is used to create a tunnel in the space between the vastus medialis and the capsule. A suture loop is pulled through the tunnel.
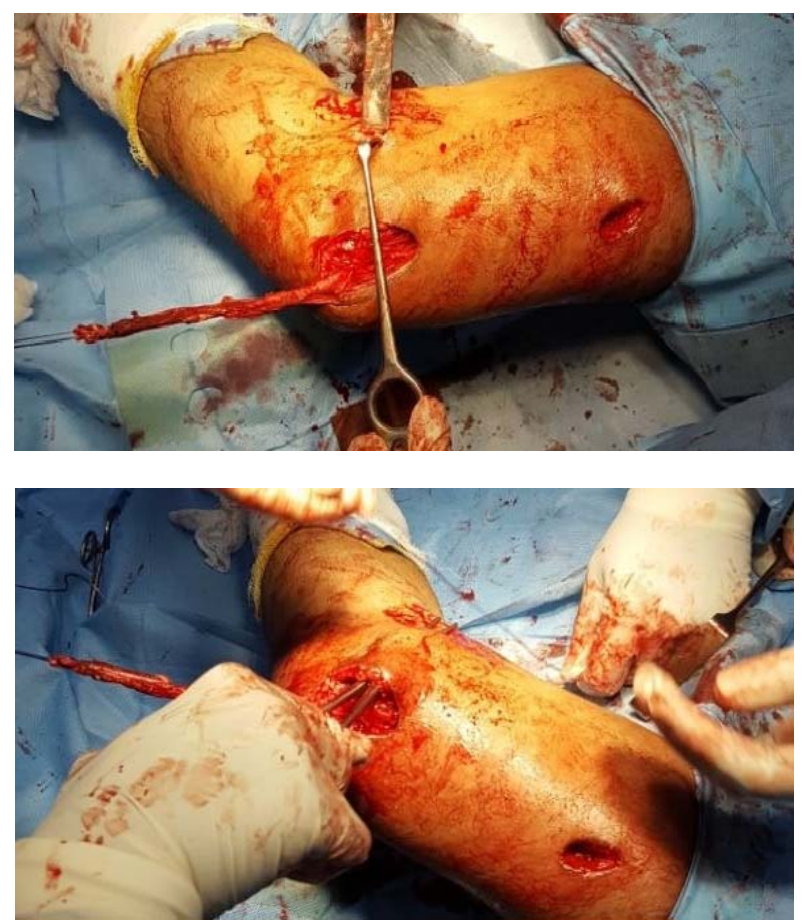

Under fluoroscopic guidance, a 2.4-mm guide pin is drilled into the insertion of the MPFL in anterolateral direction. The guide pin is over-reamed with a cannulated reamer to a depth of $30 \mathrm{~mm}$.

Fter the graft is inserted into the tunnel, knee is cycled 5 times with moderate tension on the graft. Fixation is performed with a resorbable interference screw with a similar diameter to the tunnel at 20 dgrees of knee flexion. Wound is closed in layers after achieving adequate haemo stasis.

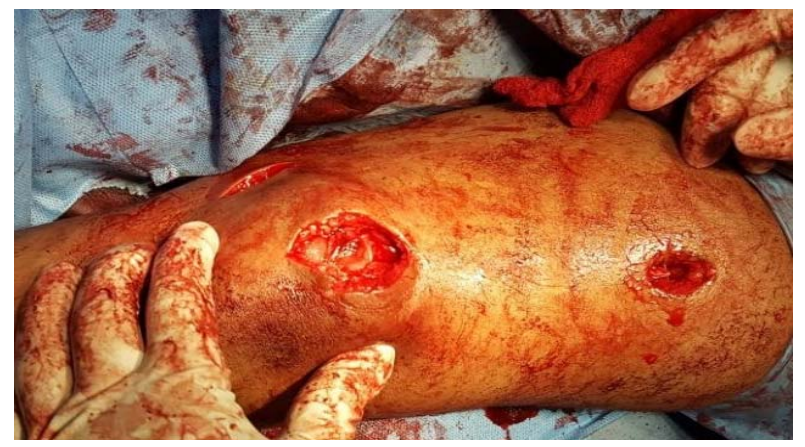

\section{Postoperative Treatment}

Knee brace is used for 2 weeks. Partial weight bearing is started 2 weeks after surgery. Full weight bearing is started thereafter. Passive range-of-motion exercises to a maximum of 90 are initiated 4th postoperative day.

Stationary cycling is started 6 weeks postoperatively. A full return to daily activities was possible by $5^{\text {th }}$ post operative month. 


\section{Results}

Out of 16 cases 9 were females and the rest males. The mean a age of cases while under going surgey was 22.5 years. $50 \%$ of cases showed 4 episodes of dislocation of patella and the rest had 3 times.3out of 16 cases were undergone previous knee surgery.10 patients had previous history of trauma.

The mean Q angle was 22.25 (16-28). One had beighton hyper mobility score greater than 5.Patellar glide test was positive in all cases. $\mathrm{J}$ sign was positive in 9 out of 16 cases. 7 out of 16 cases doesn't show the elevation of lateral edge of patella while push on the patella in order to flip lateral edge upwards with knee in extension. Apprehension test was positive in all cases. 13 out of 16 showed a positive basett's sign. Insal salvetti ratio was greater than 1.2 in 4 cases. Among all the cases 12 showed congruence angle greater than $-6$.

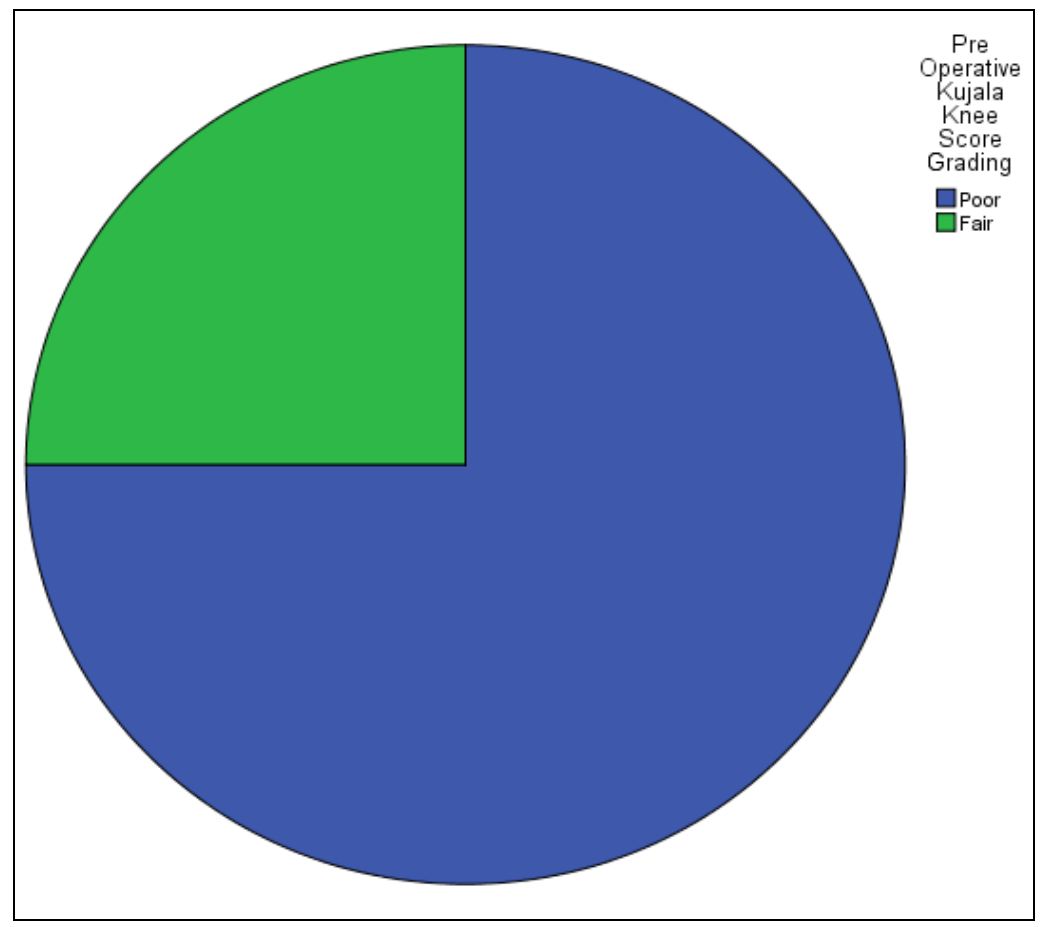

The mean Kujala knee score before surgery was 62.50 (range 56-66). $25 \%$ of cases showed poor $75 \%$ and were having fair scores. The mean Kujala score 8 months after surgery was 86 . (range 78-97) $12.5 \%$ of cases showed excellent outcome $37.5 \%$ were having good and cases with fair outcome being $50 \%$.

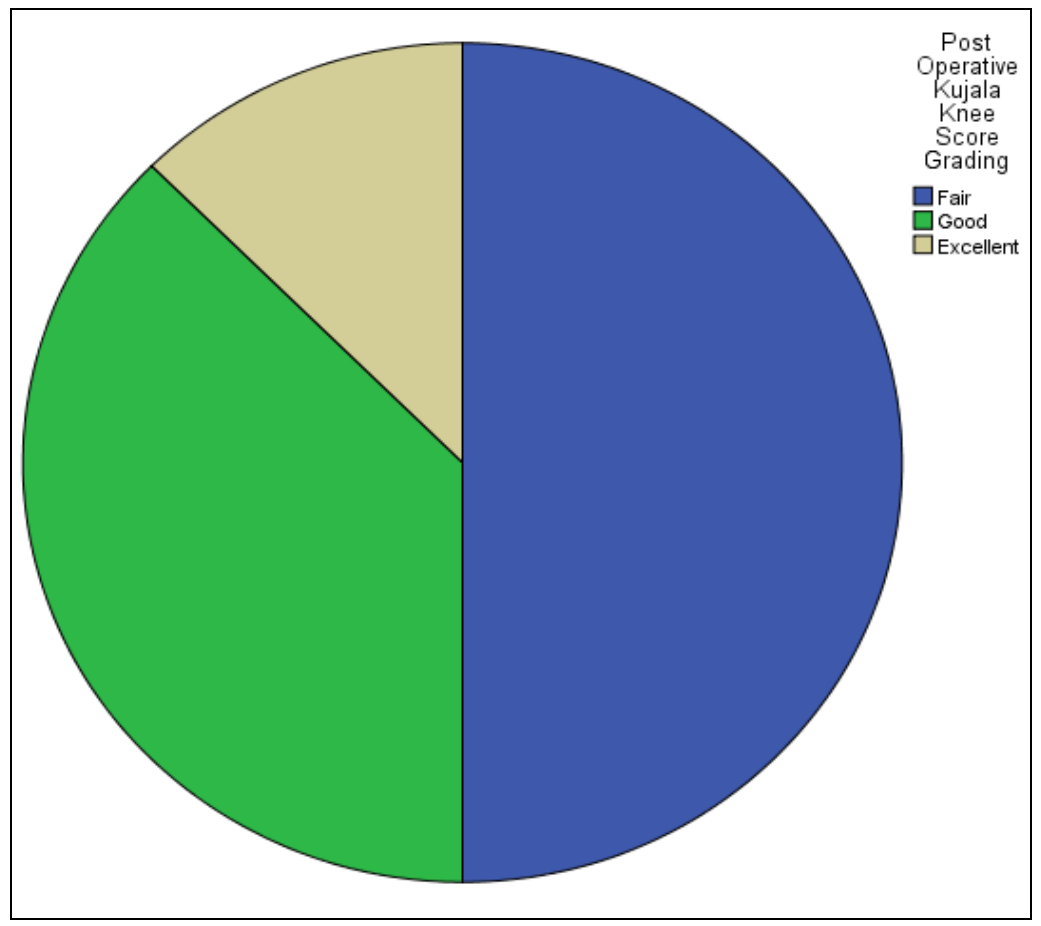

Minimally invasive quadriceps tendon graft for medial patellofemoral instability showed significant improvement in patellofemoral ligament reconstruction for chronic 

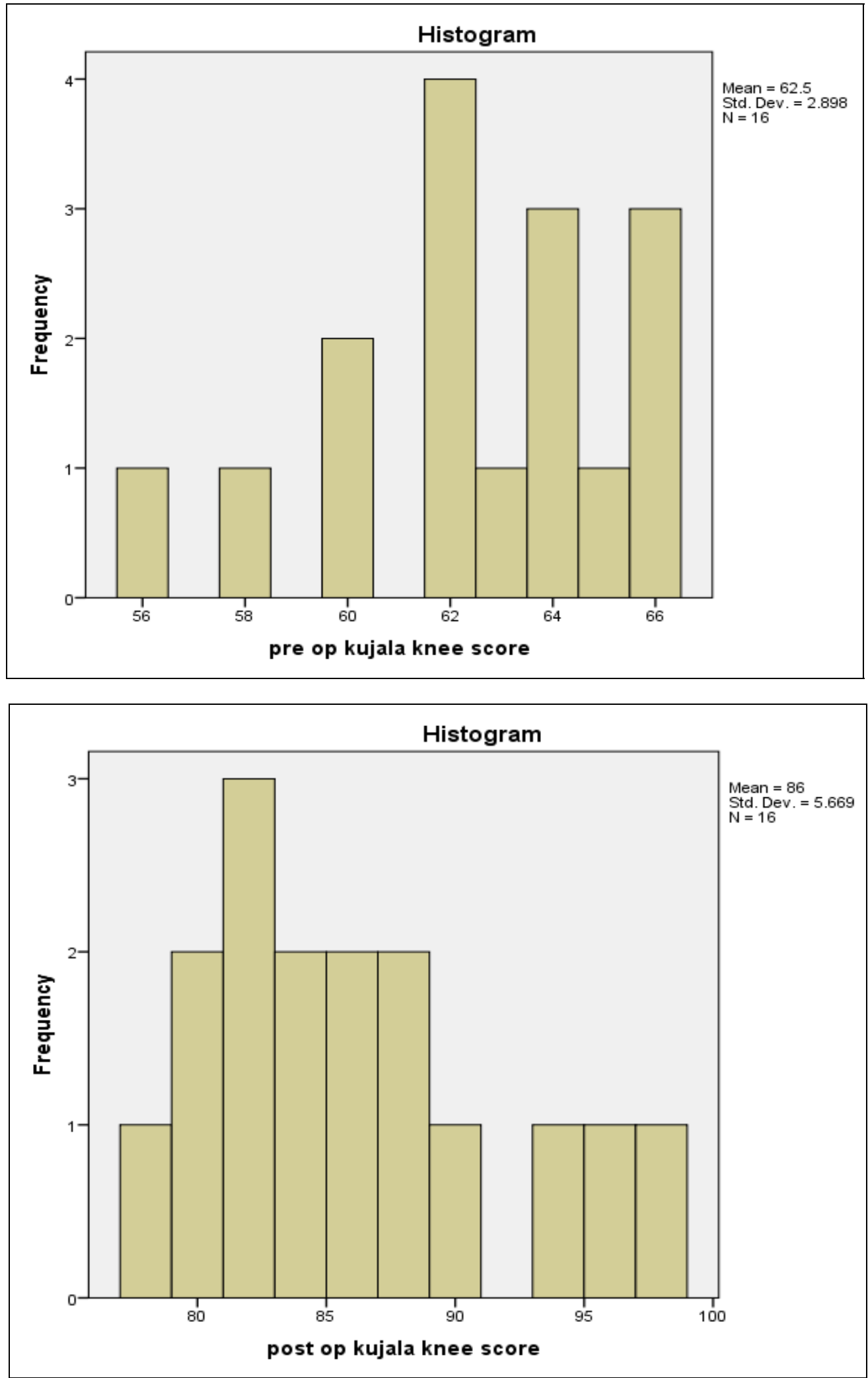

None were reported with recurrent episode during follow up of 8 months. Complication noted during surgery was graft length of $4 \mathrm{~cm}$ for only one case.

\section{Discussion}

MPFL reconstruction using a strip of Quadriceps Tendon harvested subcutaneously in the described technique had been found to be associated with good short term functional outcome. Commonly used source of graft for Medial patellofemoral ligament was harmstring. Although there is high rate of clinical success for patients with patellofemoral instability with these procedures, an overall complication rate with these techniques were $26.1 \%{ }^{[5]}$. Patellar fractures through bone tunnels and loss of knee flexion were the most common complications assosciated with these techniques. The novel technique described here avoids tunnels drilled through the patella which in turn reduces the risk of patellar fracture. With these reasons MPFL reconstruction using 
quadriceps graft is gaining popularity in treatment of patellar instability.

Noyes and Albright ${ }^{[6]}$ harvest a full thickness graft from the medial aspect of the QT, leaving it attached at the superomedial border of the patella. Goyal and Steensen et al. [7] dissect a partial-thickness graft of 10 to $12 \mathrm{~mm}$ in width from the central part of the QT.

\section{Conclusion}

The simple, easily reproducible and novel technique of minimally invasive quadriceps tendon graft for medial patellofemoral ligament reconstruction proves to be having good functional out come in chronic patellar instability

\section{Limitations}

Number of cases were less and duration of follow up was only 8 months.

Disclosure of interest authors declare that they have no conflicts of interest concerning this article

\section{References}

1. Fithian DC, Paxton EW, Stone ML et al. Epidemiology and natural history of acute patellar dislocation. Am J Sports Med. 2004; 32(5):1114-21

1. Nomura E, Inoue $M$, Kurimura $M$. Chondral and osteochondral injuries associated with acute patellar dislocation. Arthroscopy. 2003; 19(7):717-21.

2. Colvin AC, West RV. Patellar instability. J Bone Joint Surg Am. 2008; 90(12):2751-62

3. Amis AA, Firer P, Mountney J, Senavongse W, Thomas NP. Anatomy and biomechanics of the medial patellofemoral ligament The Knee. 2003; 10(3):215-20.

4. Shah JN, Howard JS, Flanigan DC, Brophy RH, Carey JL, Lattermann C. A systematic review of complications and failures associated with medial patellofemoral ligament reconstruction for recurrent patellar dislocation. Am J Sports Med. 2012; 40:1916-1923.

5. Noyes FR, Albright JC. Reconstruction of the medial patellofemoral ligament with autologous quadriceps tendon. Arthroscopy. 2006; 22:901-907.

6. Steensen RN, Dopirak RM, Maurus PB. A simple technique for reconstruction of the medial patellofemoral ligament using a quadriceps tendon graft. Arthroscopy. 2005; 21:365-370

7. Smith TO, Walker J, Russell N. Outcomes of medial patellofemoral ligamentreconstruction for patellar instability: a systematic review. Knee Surg SportsTraumatol Arthrosc. 2007; 15:1301-14.

8. Philippot R, Boyer B, Testa R, Farizon F, Moyen B. Study of patellar kinemat-ics after reconstruction of the medial patellofemoral ligament. Clin Biomech (Bristol, Avon), 2012; 27:22-6.

9. Smith TO, Davies L, Chester R, Clark A, Donell ST. Clinical outcomes of rehabilitation for patients following lateral patellar dislocation: a systematicreview. Physiotherapy. 2010; 96(4):269-81.

10. Christian Fink MD, Matjaz Veselko MD, Mirco Herbort, MD, Christian Hoser. Minimally Invasive Reconstruction of the Medial Patellofemoral Ligament Using Quadriceps Tendon Arthroscopy Techniques. 2014; 3 (3):e325-e329.

11. Kujala UM, Jaakkola LH, Koskinen SK, Taimela S, Hurme M, Nelimarkka O. Scoring of patellofemoral disorders. Arthroscopy. 1993; 9(2):159-63.

12. Crossley KM, Bennell KL, Cowan SM, Green S. Analysis of outcome measures for persons with patellofemoral pain: which are reliable and valid? Arch Phys Med Rehabil. 2004; 85(5):815-22.

13. Timm KE. Randomized controlled trial of Protonics on patellar pain, position, and function. Med Sci Sports Exerc. 1998; 30(5):665-70.

14. Nelitz M, Dornacher D, Dreyhaupt J, Reichel H, Lippacher S. The relation of the distal femoral physis and the medial patellofemoral ligament. Knee Surg Sports Traumatol Arthrosc. 2011; 19:2067-2071.

15. Christiansen SE, Jacobsen BW, Lund B, Lind $M$. Reconstruction of the medial patellofemoral ligament with gracilis tendon autograft in transverse patellar drill holes. Arthroscopy. 2008; 24:82-87.

16. Lording T, Heimstra L, Fink C, Getgood A. Strategies for reconstruction of the medial patellofemoral ligament. Op Tech Sports Med. 2015; 23(2):87-94.

17. Singhal R, Rogers S, Charalambous CP. Doublebundle medial patellofemoral ligament reconstruction with hamstring tendon autograft and mediolateral patellar tunnel fi xation: a meta-analysis of outcomes and complications. Bone Joint J. 2013; 95-b(7):900-5.

18. Panagopoulos A, van Niekerk L, Triantafi llopoulos IK. MPFL reconstruction for recurrent patella dislocation: a new surgical technique and review of the literature. Int $\mathrm{J}$ Sports Med. 2008; 29(5):359-65. 